\title{
Como a auditoria de enfermagem pode influenciar na qualidade assistencial
}

\section{How the nursing audit can influence in assistance quality}

\author{
${ }^{1}$ Lucrecia Helena Loureiro lucreciahelena@gmail.com \\ ${ }^{2}$ Leandro Martins da Costa \\ ${ }^{3}$ Vera Lúcia Marques \\ ${ }^{4}$ Clarice Mayremi Toshimitu Hoyashi
}

\begin{abstract}
RESUMO
Trata-se de estudo que tem como objetivo a auditoria de enfermagem e a qualidade assistencial· Têm como objetivos explicar de que maneira a auditoria em saúde pode influenciar para a melhoria da qualidade assistencial nas instituições de saúde e identificar quais são os itens indicadores de qualidade na auditoria. Trata-se de uma pesquisa bibliográfica sistemática de caráter descritivo e qualitativo. A seleção dos artigos foi definida em um recorte temporal, entre 2004 à 2013, sendo assim, o número total de trabalhos levantados nesse estudo foram de 19 artigos científicos, utilizados para a discussão 09 artigos de acordo com os conteúdos específicos para o estudo.Quando realizado a busca pelos artigos científicos publicados nos últimos 5 anos, notou-se que eram escassos, e portanto, foi necessário ampliar a busca de artigos para os últimos 10 anos de publicação a partir de investigações nas fontes eletrônicas do sistema Scientific Eletronic Library On-line (SCIELO), Biblioteca Virtual de Saúde (BVS), Lilacs, Google acadêmico e revistas de enfermagem indexadas. Os resultados da pesquisa mostraram que a maioria dos artigos que trataram sobre atuação da auditoria na saúde, abordou que a qualidade é de fato alcançável pelas instituições quando é presente o serviço de auditoria. A busca da qualidade é determinada pela necessidade de sobrevivência das empresas e instituições de saúde, tendo-se como resultado esperado a satisfação do cliente.
\end{abstract}

Palavras-chave: Auditoria de enfermagem. Qualidade na assistência. Informação em saúde.

\begin{abstract}
.
It is a study that aims to audit nursing and care quality. They have the objective to explain how health audit can influence to improve quality of care in health institutions and identify which items are the quality indicators in the audit. It is a systematic literature search of descriptive and qualitative. The selection of items was set in a time frame from 2004 to 2013, thus the total number of jobs raised in this study were 19 scientific articles used to discuss 09 items according to the specific content for estudo. Quando conducted the search for scientific articles published in the last five years, it was noted that they were scarce, and therefore it was necessary to expand the search for articles for the last 10 years of publication from investigations in electronic sources system scientific electronic Library On- line (SCIELO), Virtual Health Library (VHL), Lilacs, Google scholar and indexed nursing journals. The survey results showed that most of the articles that dealt on the audit in health activities, addressed that quality is actually achievable by institutions when this is the audit service. The pursuit of quality is determined by the need for survival of companies and health institutions, having as a result of expected customer satisfaction.
\end{abstract}

Keywords: Nursing auditing. Assistance quality. Health information.

1 Enfermeira Doutoranda no Programa de Enfermagem e Biociências - Universidade Federal do Estado do Rio de Janeiro- UNIRIO. Mestre em Ensino de Ciências da Saúde e Meio Ambiente, Docente da Faculdade de Enfermagem UniFOA.

2 Enfermeiro graduado na Universidade Barra Mansa.

3 Enfermeira graduada na Universidade Barra Mansa.

4 Enfermeira Mestre em Ensino de Ciências da Saúde e Meio Ambiente, Docente da Faculdade de Enfermagem UniFOA. 


\section{INTRODUÇÃO}

Conforme Pereira e Takahashi (1991) descreveram que foi nos Estados Unidos onde se iniciou a auditoria em saúde, aparecendo pela primeira vez no trabalho realizado pelo médico George Gray Ward, em 1918, quando este verificava a qualidade da assistência prestada aos pacientes por intermédio dos registros em seus prontuários.

De acordo com COREN (2001), no Brasil, a auditoria médica e de enfermagem surgiu de modo incipiente na década de 70. Desde então, tem-se ampliado a prática da auditoria em saúde, com uma progressiva absorção da mão-de-obra de enfermeiras (os). Essas atividades foram desenvolvidas pelas enfermeiras auditoras e aprovadas pelo Conselho Federal de Enfermagem através da Resolução n. 266/01.

A categoria médica e de enfermagem, possuem distintamente uma visão holística sobre os procedimentos médicos e de enfermagem implementados no tratamento e recuperação dos pacientes, o que facilita a análise de documentos procedentes para a assistência ou da assistência.

Atualmente, é na área privada onde se observa um número maior de enfermeiras auditoras, cujo conhecimento e experiência profissional são particularmente utilizados para a racionalização dos custos envolvidos na prática assistencial, atuando em instituições hospitalares ou em operadoras de planos de saúde. (MOTTA; 2003)

Demonstra-se dessa forma, que na atuação da enfermagem, há importância na utilização da auditoria como um método avaliativo, podendo contribuir para a tomada de decisões significativas no que tange a qualidade do atendimento.

O termo Qualidade ou Melhoria Contínua da Qualidade nos conceitos mais modernos é um fenômeno continuado de aprimoramento, que estabelece progressivamente os padrões, resultado dos estudos de séries históricas na mesma organização ou de comparação com outras organizações semelhantes, em busca do defeito zero - situação que, embora não atingível na prática, orienta e filtra toda ação e gestão da qualidade. É também um processo essencialmente cultural e desta forma envolve motivação, compromisso e educação dos participantes da entidade, que são assim estimulados a uma participação de longo prazo no desenvolvimento progressivo dos processos, padrões e dos produtos da entidade. (AZEVEDO; 1993)

O conhecimento e a habilidade para a implementação de cuidados são mensurados pelos pacientes por meio de avaliações que consistem em melhorias contínuas do trabalho.

A auditoria, por sua vez, é responsável por verificar o funcionamento de todo o sistema que promove o atendimento às necessidades de saúde do indivíduo, mantendo a acessibilidade e integralidade do cuidado.

De acordo com Pereira e Takahashi (1991), a auditoria em enfermagem, é uma "avaliação sistemática da qualidade da assistência de enfermagem, verificada através das anotações de enfermagem no prontuário do paciente e/ou das próprias condições deste”.

Camelo et al. (2009) citou que a auditoria em saúde , é uma área pouco investigada, mas relevante instrumento avaliativo da qualidade da assistência nas instituições públicas ou privadas, está relacionado à importância da enfermagem e sua repercussão para o cuidado prestado, associado ao controle administrativo e financeiro, uma falha qualquer nos registros ocasiona erros de cobrança, prejuízos financeiros e dificulta o faturamento do prontuário do cliente. 
Fazendo-se uma análise desde a formação profissional à atuação dos profissionais de saúde, observa-se que tal área de formação ainda é restrita, pouco divulgada nos cursos de graduação, das quais muitas vezes somente divulgada por cursos de pós-graduação, com pouca oferta de qualificação pelas instituições de ensino superior.

Ainda que lento o crescimento e a divulgação dessa especialidade, o auditor em saúde, gradativamente tem se posicionado frente às grandes organizações de saúde. Infelizmente, a percepção e a necessidade de se terem auditores numa instituição vem sendo reconhecida quando muitas vezes há problemas críticos com tendências a piorar.

O especialista em auditoria de enfermagem nas instituições de saúde utiliza seu conhecimento técnico científico, aliado a sua vivência profissional para reconhecer na organização institucional as não conformidades relacionadas à gestão e aos custos assistenciais. Também, é responsável pela avaliação dos processos de trabalhos de um modo geral, podendo contribuir para determinar o uso de indicadores de qualidade.

Segundo Pinto et a.l ( 2010) a presença do enfermeiro entre os profissionais que atuam nos serviços de auditoria em saúde denota a relação que existe entre as responsabilidades assumidas por eles na prática assistencial e o conhecimento necessário para o trabalho no campo da auditoria. Isto porque o profissional que mais atua nos procedimentos relacionados à assistência prestada ao usuário é o enfermeiro; desde o atendimento direto, até as atividades administrativas e gerenciais, é ele que confere alguma competência técnica-administrativa para lidar com o processo de produção da conta do hospital.

Partindo desse pressuposto, surgiu o interesse em realizar esse estudo pela necessidade de entender como a auditoria em enfermagem pode interferir na qualidade assistencial. Sabe-se que as instituições de saúde, de modo geral, seja privada ou pública, que possuem seus recursos humanos e materiais, num ambiente terapêutico que supostamente sejam adequados, tem a necessidade de aprimorar os processos de trabalho mediante à análise de auditoria para a promoção de melhorias no atendimento prestado.

Entretanto, sob a visão administrativa, deve haver um equilíbrio entre os custos assistenciais e o saldo positivo financeiro, para que a instituições de saúde possam realizar seus investimentos. Mas, por outro lado, os clientes tornam-se cada vez exigentes, buscando a satisfação nesses serviços. Torna-se então, um grande desafio para os auditores, o oferecimento de serviços melhores e a redução de custos hospitalares.

Conforme Luongo (2011), a auditoria em saúde tem a finalidade promover a qualidade assistencial prestada ao cliente e o controle dos custos para o adequado gerenciamento da instituição e resulta em padrões de assistência aceitáveis, satisfazendo ao cliente com a qualidade esperada e satisfazendo a instituição com o aumento da clientela à custos compatíveis, gerando lucratividade.

E para Adami (2000), a qualidade da assistência à saúde deve maximizar medidas abrangentes para o completo bem-estar do cliente, equilibrando ganhos e perdas, inerentes ao processo da atenção médico-hospitalar. Na enfermagem, a qualidade da assistência prestada aos clientes pode ser mensurada através da auditoria, um dos instrumentos internos que o serviço de enfermagem dispõe para o gerenciamento da qualidade.

Diante disso, o estudo tem como objetivos explicar através de um levantamento bibliográfico, de que maneira a auditoria em saúde pode influenciar para a melhoria da qualidade assistencial nas instituições de saúde e identificar em artigos científicos quais são os itens indicadores de qualidade da auditoria.

Emerge com isso a seguinte questão problema: Será que os enfermeiros estão preparados para o exercício da auditoria? 
Embora a prática dos enfermeiros auditores, ainda seja pouco divulgada no Brasil, a atividade deve ser exercida com competência e responsabilidade para a otimização dos recursos materiais disponíveis nos serviços de saúde, assim como, o desenvolvimento físico-estrutural e do pessoal técnico.

Kurgant (2008) , explicou que as melhorias tornam-se possíveis por meio de obtenção de conhecimento, e de capacitação dos profissionais envolvidos no processo assistencial. Além do paciente, a equipe também apresenta benefícios, sendo estes evidenciados através de reflexão de aspectos positivos e negativos, gerados a partir do desenvolvimento profissional, perante a equipe e/ou a si próprio. A participação do corpo de auditoria no planejamento das ações técnica do trabalho é uma estratégia que visa a relação custo-benefício para o paciente, as operadoras e os prestadores da saúde.

Para os enfermeiros, o embasamento de conhecimentos teóricos e práticos, aliados a experiência profissional do cotidiano é fundamental, pois permitem a eles condições de argumentação mediante de diversas situações de procedimentos clínicos e cirúrgicos a serem realizados ou realizados nos pacientes.

As experiências vivenciadas ao longo do trabalho do enfermeiro em vários setores sejam em unidade hospitalar ou clínicas/ambulatórios, em serviços públicos ou privados aumentam o saber profissional.

Além disso, faz-se necessário ainda, ser um profissional voltado para pesquisas científicas utilizando diversos instrumentos como computador, livros e artigos científicos que possam auxiliar em novas buscas de aprendizagem, possibilitando dessa forma sua atuação no processo educativo.

A auditoria, quando entendida como um processo educativo fornece subsídios para a implantação e gerenciamento de uma assistência de qualidade. Com essa ideia, espera-se que a auditoria possa dar enfoque aos problemas para nortear ações adequadas ao gerenciamento do sistema de saúde.

Segundo Scarparo \& Ferraz (2008) a auditoria de enfermagem está cada vez mais sendo inserida no mercado de trabalho, tanto na área contábil quanto na qualidade dos serviços, documentos ou processos, ao qual cumpre uma finalidade institucional voltada para o enfoque empresarial e mercadológico, mas também há uma tendência do enfoque do mercado voltado para o cliente, no que diz respeito na qualidade do produto ou serviço.

A auditoria de enfermagem tem sido uma ferramenta importante nas instituições de saúde, com a finalidade de avaliar, verificar e melhorar a qualidade da assistência, processos e custos, concentrando nos registros e anotações de enfermagem, ao qual faz parte da rotina destas empresas com o intuito de avaliar os aspectos qualitativos da assistência requerida pelo paciente, os processos internos e as contas hospitalares, possibilitando a análise das questões específicas e relativas à enfermagem (SCARPARO et al., 2009).

Toda documentação necessária, tais como prontuário médico do paciente, registros da equipe multidisciplinar, pareceres, exames e outros, fazem parte de uma coletânea de informações necessárias à avaliação da auditoria em saúde sobre as contas hospitalares. Quanto mais detalhada a informação ofertada pelas prestadoras de saúde, maior a visibilidade do atendimento realizado e a ser auditado.

Tronchin (2009) defendeu a ideia de que a qualidade em saúde possibilita a recuperação, reduzindo a probabilidade da ocorrência de eventos indesejados, de modo que a qualidade não se constitui em um atributo abstrato, pois é formada pela avaliação assistencial, apreendendo a análise da estrutura, do processo e do resultado.

A prática ideal da auditoria seria dada à devolução de informações para a equipe assistencial, com o objetivo de aperfeiçoar o cuidado prestado, demonstrando a importância do método avaliativo da auditoria no trabalho da enfermagem, com a finalidade de corrigir erros em processos vigentes. 
O prontuário na prática de enfermagem foi introduzido por Florence Nightingale, na Guerra da Criméia. Nele, Florence relatava informações a respeito dos pacientes feridos e da assistência a eles destinada, para que outros pudessem dar continuidade ao serviço. (POSSARI, 2007).

Os prontuários devem conter informações sobre o paciente incluindo o estado fisiológico e geral, psicossocial, físico e procedimentos realizados e seus resultados. Compõe, também, um meio de comunicação entres os membros da equipe, sendo indispensável qualquer outra informação sobre o paciente de qualquer outro meio (D’INNOCENZO et al., 2006).

Venturini (2008) descreveu que o processo de melhoria das anotações depende também da valorização e empenho dos profissionais em reconhecer que a Sistematização da Assistência de Enfermagem - SAE, se realizada adequadamente, pode facilitar seu trabalho e direcionar a assistência prestada ao paciente. Portanto, este não deve medir esforços para que seja aplicada com a finalidade de individualizar o cuidado, ao em vez de rotinizá-lo e/ou mecanizá-lo.

Baseado nessas informações torna-se necessário citar que a auditoria em saúde pode estar presente em várias fases do atendimento ao cliente, antes do procedimento médico ser executado, com avaliação prévia do auditor sobre a necessidade ou indicação do procedimento, durante a intervenção do procedimento médico principalmente às de alto custo ou durante a internação hospitalar para verificar necessidade de permanência hospitalar e análise pós-intervenção ou internação hospitalar.

Dessa maneira, compreende-se o motivo pelo qual o auditor funciona como uma engrenagem dentro do sistema de saúde.

De acordo com Pereira; Takahashi (1991) e Ricaldoni (2006), explanaram que o enfermeiro é a referência de sua equipe, para sanar dúvidas, auxiliar na incorporação de novas tecnologias, e por isso deve buscar na educação permanente o crescimento pessoal e profissional e contribuir para a organização do processo de trabalho, através de etapas que possam problematizar a realidade e produzir mudanças.

Dessa forma, a auditoria em enfermagem vem adquirindo novas dimensões e mostrando sua importância dentro das instituições hospitalares e operadoras de planos de saúde (MOTTA, 2003).

Há uma grande preocupação por parte das instituições de saúde em promover melhorias na qualidade assistencial e torna-se competitivos no mercado, importantes para a sobrevivência das empresas privadas, sendo assim, criam-se novas perspectivas para a auditoria em saúde e mais informação para sua equipe multidisciplinar para que haja um melhor satisfação da equipe e também do seu cliente.

Algumas dessas instituições, que se preocupam com a qualidade prestada, utilizam-se desse meio através da contratação de empresas credenciadas, para padronizar o atendimento aos clientes e alcançarem a ISO 9000 (Qualidade Total). (SCARPARO et al., 2009).

Se D’innocenzo (2006) considerou que na assistência de enfermagem existem diversos fatores que influenciam as ações desempenhadas pela equipe. Esses fatores têm base administrativa, e seguem padrões que são utilizados como análise do que foi realizado, para caso necessário, a correção de erros ou algo inadequado.

Não obstante, Matsuda (2002), afirmou que existe uma necessidade, cada vez maior, de se quantificar os produtos e para isto é necessária, por parte dos fornecedores, a elaboração de indicadores e padrões, que possibilitem a estruturação do serviço, facilitando o acompanhamento, a avaliação e o replanejamento dos processos. 
Nesse sentido, a padronização de procedimentos, modelos administrativos de diversos pensadores são adotados para obtenção do controle de custos assistenciais, aliados aos resultados de satisfação do clientes atendido.

A equipe de enfermagem, como integrante da equipe de saúde, em parceria com os auditores, deve compartilhar dados dos indicadores assistenciais de forma constante e regular, em busca de melhorias na gestão dos serviços de saúde e, conseqüentemente, contribui com a organização na busca da excelência na gestão hospitalar.

Na visão de Paulino (2009) os profissionais de enfermagem são os principais elementos humanos que contribuem para que o processo de auditoria seja executado de maneira eficiente voltada à assistência ao paciente através de um melhor controle sobre o prontuário. Infelizmente, há poucos profissionais atuando na área. Com isso, existe uma grande necessidade de orientar os profissionais de enfermagem quanto à importância de sua contribuição no processo de execução de seus serviços, bem como sobre os benefícios gerados pela auditoria.

Diante desse contexto, o presente estudo poderá contribuir para o ensino, prática e pesquisa. Para o ensino poderá colaborar para o enriquecimento do conhecimento técnico científico, assim como para a capacitação acadêmica sobre a auditoria em saúde. Na prática, poderá contribuir através de análises de dados nortearem ações de melhorias no processo de trabalho das instituições de saúde. E, na pesquisa, a divulgação através da mídia, poderá servir referência e fonte para outros estudos.

\section{METODOLOGIA}

Trata-se de uma pesquisa bibliográfica sistemática de caráter descritivo e qualitativo, constituída por artigos nacionais sobre a temática auditoria de enfermagem e a qualidade da assistência. Mas também, busca reconhecer na auditoria em saúde, um instrumento que se destina à melhoria contínua da qualidade da assistência prestada ao paciente.

Cervo, Bervian e Da Silva (2007) definiram a pesquisa bibliográfica como aquele que procura explicar um problema a partir de referências teóricas publicadas em artigos, livros, dissertações e teses.

Brevidelli e Sertório (2010) citaram que a pesquisa é descritiva quando os resultados são expressos em narrativas, descrições, figuras, declarações de pessoas, quadros esquemáticos. O significado é a preocupação essencial na abordagem qualitativa, os pesquisadores buscam detectar os significados que as pessoas dão aos fenômenos.

Para alcançar os objetivos propostos neste estudo, realizou-se uma revisão das publicações de revistas de saúde na Biblioteca Virtual da Saúde (BVS), estando nela compreendida a Scietific Eletronic Library Online - Brasil (SCIELO) e a Ciência da Saúde em Geral, que possui a seguinte base de produção científica: a Literatura Latino-americana e do Caribe em Ciências da Saúde (LILACS), utilizando-se os seguintes descritores: Auditoria de enfermagem; Qualidade na assistência; Auditoria em saúde.

Além das bases de dados citadas acima, também, buscou-se publicações eletrônicas on-line global (DEDALUS), que apresentam periódicos nacionais com artigos pertinentes ao tema abordado, sendo eles: Revista Brasileira de Enfermagem, Revista Nursing, Revista Eletrônica de Enfermagem, Paulista de Enfermagem.

Para melhorar a compreensão do estudo bibliográfico foram utilizadas outras literaturas como livros que possuíam o mesmo contexto. 
A seleção dos artigos científicos ocorreu com a exclusão de artigos estrangeiros e a apresentação do artigo somente por resumos. Os trabalhos escolhidos foram artigos científicos completos no recorte temporal entre os anos de 2004 a 2013, sendo assim, o número total de trabalhos levantados nesse estudo foram de 19 artigos científicos, utilizados para a discussão 09 artigos de acordo com os conteúdos específicos para o estudo.

Quando realizado a busca pelos artigos científicos publicados nos últimos 5 anos, notou-se que eram escassos, e portanto, foi necessário ampliar a busca de artigos para os últimos 10 anos de publicação.

Após a leitura sistemática e a compreensão dos conteúdos dos artigos científicos, alguns artigos puderam servir para fundamentar a temática, na introdução do estudo, mas também foram úteis para formalizar a discussão.

Para dar luz aos objetivos do estudo buscou-se nesses artigos o enfoque para os mesmos. Os artigos científicos foram numerados aleatoriamente de A1 a A19 para identificação durante a discussão.

A estruturação do quadro 1 é um espelho que retrata, os artigos encontrados mediante aos objetivos traçados nessa pesquisa. A partir daí realizou-se a discussão dos artigos com o raciocínio crítico de ideias sobre a temática.

\section{DISCUSSÕES DOS RESULTADOS}

Após a leitura e interpretação dos 19 artigos científicos sobre a Auditoria de enfermagem e a qualidade assistencial, foi elaborado um quadro explicativo esboçando os objetivos traçados desse estudo.

No quadro 1 foram descritos 5 artigos científicos que explicaram como a auditoria de enfermagem pode influenciar para a melhoria da qualidade assistencial e somente 4 artigos científicos identificaram quais são os itens indicadores de qualidade na auditoria.

Embora o estudo tenha envolvido 19 artigos sobre o assunto de interesse, a maioria descrevia em linhas gerais os aspectos da gestão em saúde e da auditoria sem dar enfoque necessário para responder aos objetivos da pesquisa.

Quadro 1 - Artigos pesquisados X objetivos da pesquisa.

\begin{tabular}{|c|c|c|}
\hline Identificação dos Artigos & Objetivos da Pesquisa & Autores, Títulos, Ano de publicação \\
\hline A17;A19;A13;A15;A11 & $\begin{array}{l}\text { Explicar de que maneira a auditoria de } \\
\text { enfermagem pode influenciar para a } \\
\text { melhoria da qualidade assistencial. }\end{array}$ & $\begin{array}{l}\text { SCARPARO \& FERRAZ. Auditoria em } \\
\text { enfermagem: identificando sua concepção } \\
\text { e métodos,2008. } \\
\text { VENTURINI DA, Marcon Ss. Anotações } \\
\text { de enfermagem na Unidade cirúrgica de } \\
\text { um hospital escola, } 2008 . \\
\text { PINTO et al. A prática da enfermeira em } \\
\text { auditoria em saúde, } 2010 . \\
\text { RICALDONI, Cac; SENA, Rr. Educação } \\
\text { permanente: uma ferramenta para pensar e } \\
\text { agir no trabalho de enfermagem, } 2006 . \\
\text { PAULINO, E. A. Conhecimento dos } \\
\text { Enfermeiros acerca da Auditoria } \\
\text { Hospitalar, } 2009 \text {. }\end{array}$ \\
\hline
\end{tabular}




\begin{tabular}{|l|l|l|}
\hline A16;A18;A6;A19 & $\begin{array}{l}\text { Identificar quais são os itens indicadores } \\
\text { de qualidade na auditoria. } \\
\text { SCARPARO et al., Abordagem conceitual } \\
\text { de métodos e finalidade da auditoria de } \\
\text { enfermagem, 2009. } \\
\text { TRONCHIN. Teóricos para a construção e } \\
\text { Implantação de indicadores de qualidade } \\
\text { em saúde, 2009. } \\
\text { D’INNOCENZO et al., Indicadores, } \\
\text { auditorias, certificações: ferramentas de } \\
\text { qualidade para gestão em saúde, 2006. } \\
\text { VENTURINI; Anotações de enfermagem } \\
\text { na Unidade cirúrgica de um hospital } \\
\text { escola, 2008. }\end{array}$ \\
\hline
\end{tabular}

Fonte: dos autores.

Conforme o primeiro objetivo do estudo, a auditoria em enfermagem pode influenciar para a melhoria da qualidade assistencial por intermédio da auditoria em qualidade assistencial, que verifica a qualidade da assistência prestada por meio da inspeção, avaliação e implantação de protocolos e da anotação da equipe multidisciplinar com o objetivo de analisar se o custo final do atendimento está de acordo com o atendimento prestado.

Nessa linha de raciocínio Scarparo et al. (2009), definiram que para exercer esse papel, o auditor deve ser um profissional ético, ter conhecimento técnico-científico acompanhado de todo o desenvolvimento tecnológico na saúde em geral e principalmente nas especialidades de enfermagem que audita; conhecer os estudos atuais práticos baseados em evidências; conhecer os aspectos legais que regem a profissão; desenvolver a capacidade de persuasão pela experiência anterior.

A qualidade na assistência à saúde remete a uma visão holística sobre todos os cuidados prestados aos clientes, por isso, é significativa a preocupação com os custos da assistência, pois o processo de trabalho faz parte de uma engrenagem que movimenta o setor financeiro e a forma em que o cuidado é ofertado.

É necessário pontuar que a qualidade dos serviços de saúde é avaliada pelos seus usuários e que estas, dependem dos serviços prestados. Na avaliação dos auditores em saúde, os clientes devem estar satisfeitos e os custos assistenciais devem ser reduzidos para que as empresas possam promover melhorias de diversas formas, como capacitação profissional, investimentos tecnológicos e adequação da estrutura física.

Não obstante, quando focamos qualidade, não podemos excluir o fato de que a lucratividade do negócio, não pode sobrepor à qualidade. Mas a lucratividade pode ser praticada concomitantemente às propostas de melhorias que visem minimizar eventuais falhas e o retrabalho.

Dessa forma Venturini (2008) citou a importância da informação, pois o processo de melhoria das anotações depende da valorização e empenho dos profissionais em reconhecer que a Sistematização da Assistência de Enfermagem - SAE, se realizada adequadamente, pode facilitar seu trabalho e direcionar a assistência prestada ao paciente.

Pode-se dizer que a Sistematização da Assistência de Enfermagem é um processo de cuidado com base em registros de enfermagem. Ferramenta que auxilia o entendimento da assistência prestada ao paciente desde a admissão até a alta do paciente no ambiente hospitalar. 
Segundo Pinto et al. ( 2010) a presença do enfermeiro entre os profissionais que atuam nos serviços de auditoria em saúde denota a relação que existe entre as responsabilidades assumidas por eles na prática assistencial e o conhecimento necessário para o trabalho no campo da auditoria.

Portanto necessidade de habilitação do enfermeiro auditor para realizar a auditoria agregando a vertente contábil e de qualidade. Essa é uma forte tendência a ser implementada, uma vez que instituições de saúde com custos otimizados, passam a ter subsídios financeiros para investimentos em sua estrutura (recursos humanos, tecnológicos e físicos), oferecendo, assim, suporte para as ações de melhoria da qualidade.

Também Ricaldoni (2006), explicaram que o enfermeiro é a referência de sua equipe, para sanar dúvidas, auxiliar na incorporação de novas tecnologias, e por isso deve buscar na educação permanente o crescimento pessoal e profissional e contribuir para a organização do processo de trabalho, através de etapas que possam problematizar a realidade e produzir mudanças.

A identidade profissional e à vigilância da qualidade de vida da instituição em que trabalha, fazendo cumprir sua competência profissional através de sua visão holística do ser humano durante a elaboração de medidas de desenvolver uma lógica de gestão de qualidade, não apenas pela fiscalização, ou ações estratégicas de combate ao erro ou glosas, mas principalmente por meio da educação permanente junto à equipe multidisciplinar

Na visão de Paulino (2009) os profissionais de enfermagem são os principais elementos humanos que contribuem para que o processo de auditoria seja executado de maneira eficiente voltada à assistência ao paciente através de um melhor controle sobre o prontuário. Infelizmente, há poucos profissionais atuando na área. Com isso, existe uma grande necessidade de orientar os profissionais de enfermagem quanto a importância de sua contribuição no processo de execução de seus serviços, bem como sobre os benefícios gerados pela auditoria.

A exigência do mercado em saúde que busca a qualidade acima das questões burocráticas, e para que todas as ações do enfermeiro auditor possam estar sempre do fio condutor da profissão que é o cuidado de enfermagem prestado ao paciente e assim suas ações estarão de acordo com bases teóricas necessárias para sua capacitação, pois atualmente auditoria busca qualidade da assistência com redução de custos, agregando os valores financeiros aos valores qualitativos.

De acordo com o segundo objetivo desse estudo foram identificados os indicadores de qualidade na auditoria que foram as seguintes: a) Nível de satisfação dos clientes; b) Estrutura física do atendimento; c) Qualidade do sistema.

Segundo Scarparo et al., (2009) algumas dessas instituições, que se preocupam com a qualidade prestada, utilizam-se desse meio através da contratação de empresas credenciadas, pode ser considerada um elemento essencial para mensurar a qualidade da assistência, oferecendo subsídios aos profissionais para orientar suas atividades, estimulando a reflexão individual e coletiva e nortear o processo de educação permanente

Tronchin (2009) defendeu a ideia de que a qualidade em saúde possibilita a recuperação, reduzindo a probabilidade da ocorrência de eventos indesejados, de modo que a qualidade não se constitui em um atributo abstrato, pois é formada pela avaliação assistencial, apreendendo a análise da estrutura, do processo e do resultado.

Dessa forma, a aplicação do método de auditoria em saúde beneficia os clientes que terão uma assistência de melhor qualidade através dos serviços oferecidos com mais eficácia. Os benefícios atingem também a equipe multidisciplinar que revendo as atividades desempenhadas e os resultados que se deseja alcançar obtém subsídios que estimulam a reflexão profissional. E a instituição recebe uma contribuição significativa pelo fato de verificar o alcance dos seus objetivos, constituindo base para prováveis mudanças internas. 
D’innocenzo et al. (2006) enfocou que na assistência de enfermagem existem diversos fatores que influenciam as ações desempenhadas pela equipe. Esses fatores têm base administrativa, e seguem padrões que são utilizados como análise do que foi realizado, para caso necessário, a correção de erros ou algo inadequado.

Desta forma os registros em prontuário representam um indicador de qualidade da atenção ofertada, assim como uma ferramenta para avaliar a necessidade de educação permanente, além de se constituir em instrumento para respaldo ético e legal aos profissionais responsáveis pelo cuidado,assim como ao paciente.Ela é um instrumento essencial de diminuição de glosas e aumento do faturamento hospitalar, bem como um espaço especial para rever a produção do cuidado pela equipe de enfermagem

Venturini (2008) enfatizou que o processo de melhoria das anotações depende também da valorização e empenho dos profissionais de saúde.

Importante destacar que as anotações devem servir como instrumento no processo de tomada de decisão e para tanto devem refletir com clareza e objetividade o estado clínico dos pacientes, bem como as ações e procedimentos realizados e um indicador de qualidade da assistência relevante e essencial para subsidiar do planejamento das ações, bem como sua implementação, gerenciamento e avaliação qualitativa dos resultados obtidos, visto que este processo ocorre de forma concomitante e inter-relacionada.

\section{CONSIDERAÇÕES FINAIS}

Atualmente, a exigência em qualidade no atendimento ao paciente tem levado a uma atenção especial por parte das organizações e profissionais que prestam o serviço em saúde.

A partir daí, passa a ser uma preocupação das instituições de saúde em promover melhorias de ordem estrutural, em recursos humanos e materiais, no que concerne não só a satisfação da clientela, mas, a redução de custos com metas ousadas para novos investimentos, mantendo-se dentro de um mercado competitivos.

A atuação da auditoria influencia na qualidade de serviços de saúde pelo fato de que a visão dos auditores estará voltada para os processos de trabalho mais seguros com foco na satisfação dos clientes internos e externos, assim como lucratividade do negócio, minimizando eventuais falhas e o retrabalho.

Há uma grande perspectiva de mudança na configuração atual da auditoria em saúde que se deve, principalmente, às exigências do mercado, que busca desenvolver uma lógica de gestão e qualidade. Entretanto, percebeu-se no desenvolvimento desse estudo que os profissionais de saúde ao exercerem suas atividades técnicas, muitas vezes, possuem dificuldades em realizarem os registros necessários à avaliação da auditoria, sendo este um dos fatores que podem impedir a análise desse profissional em questão.

A partir desse entendimento, pode dizer que numa análise geral, os profissionais de saúde, também auditores em saúde, ainda não estão totalmente preparados para o exercício da auditoria, considerando que a formação dos profissionais ainda possuem lacunas no processo de ensino que precisam ser repensados.

A ideologia centrada na auditoria em saúde propõe-se a fusão entre a racionalização dos custos e qualidade dos serviços dos profissionais de saúde. Novos estudos nessa área, poderão ofertar crescimento e aperfeiçoamento à formação de novos auditores, pois a auditoria em saúde é uma tendência no mercado. 


\section{REFERÊNCIAS}

ADAMI, Nilce Piva. Melhoria da qualidade nos serviços de enfermagem. Acta Paul Enferm 2000; 13(esp-pt I): 190-6. AZEVEDO, A.C. Indicadores de qualidade e produtividade em serviços de saúde. Rev Ind Qual Produt IPEA 1993. BREVIDELLI, Maria Meimei; SERTÓRIO, Sonia Cristina Masson. Trabalho de Conclusão de Curso - Guia Prático para Docentes e Alunos da Área da Saúde. 4 ed. rev. Atual. e ampl. São Paulo: Iátria, 2010.

CAMELO, Silvia Helena Henriques; PINHEIRO, Aline.; CAMPOS, Domitila.; OLIVEIRA,Tatiana Lentz. Auditoria de enfermagem e a qualidade da assistência à saúde: uma revisão da literatura. Revista Eletrônica de Enfermagem, v.11, 2009. Disponível em:<http://www.fen.ufg.br/revista/v11/n4/v11n4a28.htm.>.

CERVO, Amado L.; BERVIAN, Pedro A.; DA SILVA, Roberto. Metodologia Científica. 6 ed. São Paulo: Pearson Prentice Hall, 2007

Conselho Federal de Enfermagem (COFEN). Resolução n. 266, de 25 de outubro de 2001. Aprova atividades de enfermeiro auditor [Legislação na Internet]. Brasilia; 2001. Disponível em: <http://www.portalcofen.gov.br/2007/materias.asp>.

D’INNOCENZO, Maria. (Coord.). Indicadores, auditorias, certificações: ferramentas de qualidade para gestão em saúde. São Paulo: Martinari, 2006.

KURCGANT, Paulina. et al. Gerenciamento em enfermagem. Rio de Janeiro: Guanabara Koogan, 2005.

LUONGO, Jussara (org). Gestão de qualidade em saúde. 1 ed. São Paulo: Rideel, 2011.

MATSUDA, Laura Misue. Satisfação profissional da equipe de enfermagem na UTI - adulto: perspectivas de gestão para a qualidade da assistência [tese]. Ribeirão Preto: Escola de Enfermagem de Ribeirão Preto, Universidade de são Paulo; 2002. Disponível em: . Acessado em: 24 de Abril de 2014.

MOTTA, Ana Letícia Carnevalli. Auditoria de enfermagem nos hospitais e operadoras de planos de saúde. $3^{a}$ ed. São Paulo: Iátria; 2003.

PAULINO, E. A. Conhecimento dos enfermeiros acerca da auditoria hospitalar. João Pessoa. 2009. Disponível em: $\leq$ www.portaleducacao.com.br $>$. Acessado em: 04 de maio de 2014.

PEREIRA, L.L, TAKAHASHI ,Regina Toshie. Auditoria em enfermagem. In.: Kurcgant P, organizadora. Administração em Enfermagem. São Paulo: EPU, 1991. p. 215 -22.

PINTO, Karina Araújo, MELO, Cristina Maria Meira de. A prática da enfermeira em auditoria em saúde. Revista Esc. Enfermagem USP [série internet]. 2010 sep. [citado em 20 jun. 2011]; 44(3): 671-678. Disponível em: URL: $<$ http://www.scielo.br/scielo $>$.

POSSARI, João Francisco. Prontuário do Paciente e os Registros de Enfermagem. 2. ed. São Paulo: Iátria, 2007.

RICALDONI, Carlos Alberto Caciquinho; SENA, Roseni Rosângela. Educação permanente: uma ferramenta para pensar e agir no trabalho de enfermagem. Rev Latino-am Enfermagem. 2006; 14(6): 837-42.

SCARPARO, Ariane Fazzolo. et al. Abordagem conceitual de métodos e finalidade da auditoria de enfermagem. Rev. Rene, Fortaleza, v.10, n.1, p.1-165, jan./mar.2009.

SCARPARO, Ariane Fazzolo; FERRAZ, Clarice Aparecida. Auditoria em enfermagem: identificando sua concepção e métodos. Rev Bras Enferm. 2008; 61(3): 302-05. 
TRONCHIN, Daisy Maria Rizatto; MELLEIRO, Marta Maria; KURCGAN, Paulina; GARCIA, Andressa Nicole; GARZIN, Ana Claudia Alcântara. subsídios teóricos para a construção e implantação de indicadores de qualidade em saúde. Revista Gaúcha de Enfermagem. Porto Alegre.

VENTURINI Daniele Aparecida, MARCON, Sônia Silva. Anotações de enfermagem na unidade cirúrgica de um hospital escola. Rev. Bras. Enferm. Brasília, v. 61, n.5, outubro 2008. Disponível em: URL: $\langle$ http://www.scielo.br/scielo $>$. 